\title{
Cartan-Kuranishi's prolongation of differential systems combined with that of Lagrange and Jacobi
}

By

\author{
Michihiko Matsuda
}

\section{§o. Introduction}

There exist differential systems which can not be prolonged to an involutive system by Kuranishi's standard prolongation, although they have a solution. A simple example was given by Kuranishi himself in [8]. We shall construct here a partial prolongation, combining Cartan-Kuranishi's standard prolongation and Lagrange-Jacobi's classical prolongation. Applying our partial prolongation, we can prolong any differential system to an involutive system if it has a solution.

Let $(M, N ; \pi)$ be a real analytic fibered manifold and let $J^{\prime}(M$, $N ; \pi)$ be the space of $l$-jets. A subsheaf of ideals (which is locally finitely generated) in $\mathcal{O}\left(J^{l}\right)$, the sheaf of germs of real analytic functions defined on $J^{l}(M, N ; \pi)$, is called a system of differential equations of order $l$ on $N$. We consider here systems of differential equations of the most general type which may be non-linear and overdetermined in general.

Roughly speaking, a differential system is said to be involutive, if its general solution can be obtained by solving successively equations of Cauchy-Kowalevsky's type.

As to exterior differential systems, E. Cartan characterized involutive systems in [2]. M. Kuranishi constructed the standard prolongation of exterior differential systems in [8]. He gave in [8] and [9] a necessary and sufficient condition under which a system can be 
prolonged to an involutive system by the standard prolongation.

Lagrange considered systems of linear differential equations of the first order with one unknown function. He showed that every such system can be prolonged either to an involutive system of the first order or to an incompatible system (see [4]). Generalizing the method of Lagrange, Jacobi proved that every system of non-linear differential equations of the first order with one unknown function can be prolonged either to an involutive system of the first order or to an incompatible system (see [4]).

E. Cartan showed in [3] that any exterior differential system with two independent variables can be prolonged to an involutive system if it has a solution. He also conjectured in [3] that any exterior differential system with more than two independent variables can be prolonged to an involutive system if it has a solution.

J. A. Schouten and W. v. d. Kulk obtained in [14] the theorem of prolongation on exterior differential systems of the special type.

H. H. Johnson treated in [7] certain types of differential systems which are prolonged to an involutive system by Kuranishi's standard prolongation.

Recently M. Kuranishi characterized involutive systems of differential equations in [10]. He gave a clear proof of his prolongation theorem on systems of differential equations also in [10].

We shall compare Kuranishi's prolongation theorem with the classical theorem of Lagrange and Jacobi. Let us consider a system of differential equations $\Phi$ of the first order with one unknown function. Then it can be proved that, if $\Phi$ is not involutive, it can not be prolonged to an involutive system by the standard prolongation. Hence Kuranishi's prolongation theorem does not contain Lagrange-Jacobi's theorem as a special case.

Roughly speaking, we say that a system of differential equations $\Phi$ of order $l$ is quasi-involutive, if $p \Phi$, the prolongation of $\Phi$, contains a system $\left\{\phi_{1}, \cdots, \phi_{r}\right\}$ of functions defined on $J^{\prime}(M, N ; \pi)$ with the following property: $\Phi$ is involutive if and only if $\Phi$ contains all $\phi_{i}(1 \leqq$ 
$i \leqq r)$

From this point of view, it follows from Kuranishi's prolongation theorem that every system of differential equations can be prolonged to a quasi-involutive system of higher order by the standard prolongation, if it has a solution.

Generalizing the method of Lagrange and Jacobi, we shall define the prolongation of the same order for every system of differential equations of order $l$. Let $p_{0} \Phi$ be the set of all functions defined on $J^{l}(M, N ; \pi)$ that are contained in $p \Phi$. Then $p_{0} \Phi$ is a subsheaf of ideals in $\mathcal{O}\left(J^{l}\right)$, which contains $\Phi$. We call $p_{0} \Phi$ the prolongation of the same order of $\Phi$.

Also we shall call the subsheaf of ideals $\bigcup_{n=1}^{\infty} p_{0}^{n} \Phi$ in $\mathcal{O}\left(J^{l}\right)$ the $p$ closure of $\Phi$. Here $p_{0}^{n} \Phi$ is defined by $p_{0}^{n} \Phi=p_{0}\left(p_{0}^{n-1} \Phi\right)$ inductively. By this definition the theorem of Lagrange and Jacobi can be expressed in the following form: if $\Phi$ is a system of differential equations of the first order with one unknown function, then the $p$-closure of $\Phi$ is either involutive or incompatible. The success of Lagrange and Jacobi results from the fact that every system is quasi-involutive in their case.

The algebraic aspect of Kuranishi's prolongation theorem was described in a purely algebraic theorem by V. W. Guillemin, I. M. Singer and S. Sternberg in [5] and [15]. The theorem was conjectured first by them and was proved by J.-P. Serre (see Appendix in [5]). In his proof, J.-P. Serre clarified the relation between vanishing of Spencer's cohomologies and involutiveness, applying a theorem on commutative algebra in [1].

We shall combine the prolongation of Cartan and Kuranishi with that of Lagrange and Jacobi in the following way. For a given system of differential equations $\Phi$ of order $l$, let $\Psi_{0}$ be the $p$-closure of $\Phi$. For every integer $n$ we inductively define $\Psi_{n}$ as the $p$-closure of $p \Psi_{n-1}$. Then we have the sequence of systems $\left\{\Psi_{0}, \Psi_{1}, \Psi_{2}, \cdots \cdots\right\}$ with the following property: for every $n, p_{0} \Psi_{n}$ and $p \Psi_{n}$ are contained in $\Psi_{n}$ and $\Psi_{n+1}$ respectively. 
We say that a system $\Psi$ of differential equations is p-closed, when $\Psi$ contains $p_{0} \Psi$. Then every system $\Psi_{n}$ above constructed for $\Phi$ is $p$ closed. We shall prove in Theorem 1 that a system $\Psi$ is involutive if and only if $\Psi$ is $p$-closed and quasi-involutive.

Applying the theorem of Kuranishi, Guillemin, Singer, Sternberg and Serre, we can prove that the system $\Psi_{n}$ above constructed for $\Phi$ is quasi-involutive for sufficiently large $n$, provided it is compatible. Since every $\Psi_{n}$ is $p$-closed, we see in Theorem 2 that $\Psi_{n}$ is involutive for such sufficiently large $n$. Hence we can prolong every system $\Phi$ either to an involutive system or to an incompatible system.

As to prolongation of G-structures, N. Tanaka recently constructed in [19] the partial prolongation. He gave an application, proving finiteness of the automorphism groups of certain G-structures which are not of finite type. Our construction of the partial prolongation was motivated by Tanaka's construction of his prolongaiton.

As to prolongation of systems of linear differential equations, fruitful results are being obtained by D. C. Spencer, M. Kuranishi, D. G. Quillen, W. J. Sweeney, C. Buttin, H. Goldschmidt and others. A part of their results has been published (see [11], [13], [16], [17], [18]).

Their results are being obtained in the category of infinite differentiability. However, we discuss the problem here in the category of real analyticity. Also we discuss here local existence of solutions.

The author wishes to express his sincere gratitude to Professor M. Kuranishi, Professor T. Nagano, Professor S. Nakano, Professor D. C. Spencer and Professor N. Tanaka for their kind advices and encouragement.

\section{$\S 1$. Systems of differential equations}

Let $\mathcal{O}\left(J^{l}\right)$ be the sheaf of germs of real analytic functions defined on $J^{l}(M, N ; \pi)$ and let $\Phi$ be a subsheaf of ideals in $\mathcal{O}\left(J^{l}\right)$. Here we say that $\Phi$ is a system of differential equations of order $l$. We do not assume that $\Phi$ is locally finitely generated. The following Proposi- 
tion 1 explains what happens if we do not assume that $\Phi$ is locally finitely generated.

A point $X$ in $J^{l}(M, N ; \pi)$ is called an integral point of $\Phi$, if every $\varphi$ in $\Phi_{X}$ vanishes at $X$. Let $\bar{\Phi}$ be the set of all $\varphi$ in $\mathcal{O}\left(J^{l}\right)$ such that there exists a domain $\mathcal{U}_{0}$ with the property that $\varphi$ is defined over $\mho_{0}$ and vanishes on $U_{0} \cap I \Phi$. Here $I \Phi$ is the set of all integral points of $\Phi$. Then $\bar{\Phi}$ is a subsheaf of ideals in $\mathcal{O}\left(J^{l}\right)$, which contains $\Phi$. We have the identity $I \Phi=I \bar{\Phi}$. Hence the system $\bar{\Phi}$ has the following property: for an arbitrary domain $\mathcal{U}$, any section over $\mathcal{U}$ which vanishes on $\vartheta \cap I \bar{\Phi}$ belongs to $\bar{\Phi}$.

Proposition 1. For any domain $\mathcal{U}_{0}$ in $J^{\prime}(M, N ; \pi)$ containing an integral point of $\bar{\Phi}$, there exists in $\mathcal{U}_{0}$ an integral point $X_{0}$ around which $\bar{\Phi}$ is finitely generated on $I \bar{\Phi}$.

Proof. For every point $X$ on $\bigcup_{0} \cap I \bar{\Phi}$, we define $s(X)$ by

$$
s(X)=\operatorname{dim}\left\{d \varphi ; \varphi \in \bar{\Phi}_{X}\right\} .
$$

We assume that the function $s(X)$ attains the maximum $s$ at $X_{0}$. We can take a coordinate system $\left(\varphi_{1}, \cdots, \varphi_{s}, u_{1}, \cdots, u_{t}\right)$ around $X_{0}$ choosing $\varphi_{i}$ in $\bar{\Phi}_{X_{0}}(1 \leqq i \leqq s)$. Let $X_{1}$ be an integral point which belongs to a sufficiently small neighbourhood of $X_{0}$. For every $\varphi$ in $\bar{\Phi}_{X_{1}}$, there exists a function $\psi$ defined on a neighbourhood $\mathcal{V}_{1}$ of $X_{1}$ which satisfies the congruence

$$
\begin{aligned}
& \varphi\left(\varphi_{1}, \cdots, \varphi_{s}, u_{1}, \cdots, u_{t}\right) \\
\equiv & \psi\left(u_{1}, \cdots, u_{t}\right) \bmod \left(\varphi_{1}, \cdots, \varphi_{s}\right) .
\end{aligned}
$$

Since the function $\psi$ belongs to $\bar{\Phi}_{X_{1}}$, we have the identity $d \psi=0$ at every point on $\vartheta_{1} \cap I \bar{\Phi}$. Hence every derivative $\frac{\partial \psi}{\partial u_{k}}$ vanishes on $\vartheta_{1} \cap I \bar{\Phi}$ and belongs to $\bar{\Phi}_{X_{1}}$.

It follows also that every derivative $\frac{\partial^{m} \psi}{\partial u_{k 1} \cdots \partial u_{k_{m}}}$ of higher order belongs to $\bar{\Phi}_{X_{1}}$ and vanishes at $X_{1}$. Since the function $\psi$ is real analytic at $X_{1}$, it vanishes identically. Hence we have the congruence $\varphi \equiv 0 \bmod \left(\varphi_{1}, \cdots \varphi_{s}\right)$ for every $\varphi$ in $\bar{\Phi}_{X_{1}}$. This proves the proposition.

An integral point $X$ is called an ordinary integral point, if $\Phi=0$ 
is a regular local equation of $I \Phi$ around $X$.

Let $\rho_{l-1}^{l}$ be the projection from $J^{l}(M, N ; \pi)$ onto $J^{l-1}(M, N ; \pi)$ defined by

$$
\rho_{l-1}^{l}\left(j_{x}^{l}(f)\right)=j_{x}^{l-1}(f) .
$$

Then for every pair of $X$ and $\bar{X}=\rho_{l-1}^{l} X$, we have the injection $i$ from $\mathcal{O}_{\bar{X}}\left(J^{l-1}\right)$ into $\mathcal{O}_{X}\left(J^{l}\right)$ defined by

$$
i \varphi=\varphi \circ \rho_{l-1}^{l} .
$$

We identify $\mathcal{O}_{\bar{X}}\left(J^{l-1}\right)$ and its image by $i$ in $\mathcal{O}_{x}\left(J^{l}\right)$.

Let $\tau$ be the natural projection from $T_{X}^{*}\left(J^{l}\right)$ onto $T_{X}^{*}\left(J^{l}\right) /$ $\operatorname{Im}\left(\pi \circ \rho_{0}^{l}\right)^{*}$. We say that $\Phi$ is compatible at $X_{0}$ if $\Phi_{X_{0}}$ is generated by such functions $\varphi_{1}, \cdots, \varphi_{s}$ that $\tau d \varphi_{1}, \cdots, \tau d \varphi_{s}$ are independent at $X_{0}$ and if $X_{0}$ is an integral point of $\Phi$.

A real analytic mapping $f$ from a domain $U$ in $N$ to $M$ which satisfies $\pi \circ f=$ identity is called a solution of $\Phi$, if $j_{x}^{l}(f)$ is an integral point of $\Phi$ for every point $x$ in $U$.

Proposition 2. Let $X_{0}$ be an ordinary integral point of $\Phi$. If a solution $f$ passes through $X_{0}$, then $\Phi$ is compatible at $X_{0}$.

Proof. We choose such functions $\varphi_{1}, \cdots, \varphi_{s}$ in $\Phi_{X_{0}}$ that $\tau d \varphi_{1}, \cdots$, $\tau d \varphi_{s}$ are independent at $X_{0}$, where $s=\operatorname{dim}\left\{\tau d \varphi ; \varphi \in \Phi_{X_{0}}\right\}$.

We take a coordinate system $\left(\varphi_{1}, \cdots, \varphi_{s}, u_{1}, \cdots, u_{r}, x_{1}, \cdots, x_{n}\right)$ around $X_{0}$ with the property that $\tau d \varphi_{1}, \cdots, \tau \varphi_{s}, \tau d u_{1}, \cdots, \tau d u_{r}$ are independent at $X_{0}$ and every $x_{i}$ belongs to $\mathcal{O}(N)$. For every $\varphi$ in $\Phi_{X_{0}}$ there exists a function $\psi$ which satisfies the congruence

$$
\varphi\left(\varphi_{i}, u_{j}, x_{k}\right) \equiv \psi\left(u_{j}, x_{k}\right) \bmod \left(\varphi_{1}, \cdots, \varphi_{s}\right) .
$$

Since $\tau d \psi_{r}=0$ at $X_{0}$, for every $h(1 \leqq h \leqq r)$ we have

$$
\frac{\partial \psi}{\partial u_{h}}\left(u_{j}^{0}, x_{k}^{0}\right)=0
$$

at $X_{0}$. On the other hand we have the identity

$$
\psi\left(u_{j}\left(j_{i}^{l}(f)\right), x_{k}\right)=0
$$

from which for every $i(1 \leqq i \leqq n)$ we have 


$$
\frac{\partial \psi}{\partial u_{h}}\left(u_{j}^{0}, x_{k}^{0}\right) \frac{\partial u_{h}\left(j_{x}^{l}(f)\right)}{\partial x_{i}}+\frac{\partial \psi}{\partial x_{i}}\left(u_{\jmath}^{0}, x_{k}^{0}\right)=0
$$

at $X_{0}$. Hence for every $i(1 \leqq i \leqq n)$ we get

$$
\frac{\partial \psi}{\partial x_{i}}\left(u_{j}^{0}, x_{k}^{0}\right)=0
$$

and we have the identity $d \psi_{r}=0$ at $X_{0}$. Since $X_{0}$ is an ordinary integral point, $\Phi_{X_{0}}$ is generated by $\varphi_{1}, \cdots, \varphi_{s}$.

\section{\$2. Prolongation of systems of differential equations}

Let $\Phi$ be a system of differential equations of order $l$. M. Kuranishi defined in [10] the prolongation of $\Phi$ in the following way. Let $\xi$ be a vector field on $N$. To every function $\varphi$ in $\mathcal{O}\left(J^{l}\right)$ we associate the function $\varphi_{\xi}$ in $\mathcal{O}\left(J^{l+1}\right)$ defined by

$$
\varphi_{\xi}\left(j_{x}^{l+1}(f)\right)=\xi\left(\varphi\left(j_{x}^{l}(f)\right)\right) .
$$

Let $p \Phi$ be the subsheaf of ideals in $\mathcal{O}\left(J^{l+1}\right)$ generated by $\Phi$ and $\varphi_{\xi}$, where $\varphi$ and $\xi$ vary over all elements of $\Phi$ and all vector fields on $N$ respectively. M. Kuranishi called $p \Phi$ the prolongation of $\Phi$. Let $\left(x_{i}\right.$, $\left.y_{\alpha}, p_{\alpha}^{i}, \cdots, p_{\alpha}^{i_{1} \cdots t_{l} l}\right)$ be a coordinate system around $X$, where $p_{\alpha}^{i_{1} \cdots t_{k}}\left(j_{x}^{l}(f)\right)$ $=\frac{\partial^{k} f_{\alpha}}{\partial x_{i_{1}} \cdots \partial x_{i_{k}}}$. If $\Phi_{X}$ is generated by $\varphi_{k}(1 \leqq k \leqq r)$, then $(p \Phi)_{\tilde{X}}$ is generated by $\Phi$ and $\partial_{\sharp \varphi_{k}}^{l}(1 \leqq k \leqq r, 1 \leqq i \leqq \operatorname{dim} N)$ for every $\widetilde{X}$ in $\left(\rho_{l}^{l+1}\right)^{-1} X$. Here $\partial_{\sharp}^{i} \varphi_{k}$ is the function defined by

$$
\hat{\partial}_{\sharp}^{i} \varphi_{k}=\frac{\partial \varphi_{k}}{\partial x_{i}}+\frac{\partial \varphi_{k}}{\partial y_{\alpha}}-p_{\alpha}^{i}+\cdots+\frac{\partial \varphi_{k}}{\partial p_{\alpha}^{i_{1} \cdots l^{\prime} l}} p_{\alpha}{ }^{i_{1} \cdots i^{\prime}} .
$$

Generalizing the method of Lagrange and Jacobi, we define $p_{0} \Phi$, the prolongation of the same order of $\Phi$, as the sheaf associated to the presheaf $\{\mathscr{U} \rightarrow \mathscr{G}(\mathcal{Q})\}$. Here $\mathscr{L}(\mathcal{U})$ is the set of all elements of $\Gamma(\mathcal{U})$ that are contained in the $\Gamma(\widetilde{\mathcal{Q}})$-module generated by $\Phi$ and $\partial_{\sharp} \Phi$, where $\Gamma(\mathcal{U})$ and $\Gamma(\widetilde{\mathcal{U}})$ are the rings of all sections over $\mathcal{U}$ and $\left(\rho_{l}^{l+1}\right)^{-1} \mathcal{U}$ respectively. Then $p_{0} \Phi$ is a subsheaf of ideals in $O\left(J^{l}\right)$, which contains $\Phi$.

We say that $\Phi$ is $p$-closed at $X$, when $\Phi_{X}$ contains $\left(p_{0} \Phi\right)_{X}$. 
Following [10], we define the subspace $C_{X}(\Phi)$ of $Q_{X}\left(J^{l}\right)$ for every integral point $X$ by

$$
C_{X}(\Phi)=\left\{\mathscr{X} \in Q_{X}\left(J^{l}\right) ; \mathscr{X}(\varphi)=0 \text { for all } \varphi \in \Phi_{X}\right\},
$$

where $Q_{X}\left(J^{l}\right)=\operatorname{Ker}\left(d \rho_{l-1}^{l}\right)$. Also we define $p\left(C_{X}(\Phi)\right)$, the prolongation of $C_{X}(\Phi)$, by

$$
p\left(C_{X}(\Phi)\right)=C_{X}(\Phi) \otimes T_{x}^{*}(N) \cap Q_{X}(M) \otimes S^{l+1}\left(T_{x}^{*}(N)\right),
$$

where $Q_{X}(M)=\operatorname{Ker}\left(d_{\pi}\right)$. Then we have the identity

$$
C_{\widetilde{X}}(p \Phi)=p\left(C_{X}(\Phi)\right)
$$

for every $\widetilde{X}$ in $\left(\rho_{l}^{l+1}\right)^{-1} X \cap I(p \Phi)$.

Proposition 3. Let $X_{0}$ be an ordinary integral point of $\Phi$ which satisfies the following two conditions (i) and (ii):

(i) $\Phi$ is p-closed at $X_{0}$.

(ii) $\operatorname{dim} p\left(C_{X}(\Phi)\right)=$ constant on a neighbourhood of $X_{0}$ in IФ.

Then there exists such a neighbourhood $\mathcal{U}_{0}$ of $X_{0}$ that $\left(\widetilde{C}_{0}\right.$, $\left.\mathcal{V}_{0} ; \rho_{l}^{l+1}\right)$ forms a fibered manifold, where $\widetilde{\mathcal{V}}_{0}$ and $C V_{0}$ are $\left(\rho_{l}^{l+1}\right)^{-1} \mathcal{U}_{0}$ $\cap I(p \Phi)$ and $\mathcal{U}_{0} \cap I \Phi$ respectively.

Proof. We take a system of generators $\left\{\varphi_{1}, \cdots, \varphi_{s}\right\}$ of $\Phi_{X_{0}}$, where $s=\operatorname{codim} I \Phi$.

Let $\left\{\tilde{\varphi}_{k} ; 1 \leqq k \leqq n s\right\}$ be an arrangement of $\left\{\partial_{*}^{r} \varphi_{i} ; 1 \leqq r \leqq n, 1 \leqq i \leqq s\right\}$, where $n=\operatorname{dim} N$. And let $\left\{\widetilde{p}_{h} ; 1 \leqq h \leqq m\left(\begin{array}{c}l+n \\ l+1\end{array}\right)\right\}$ be an arrangement of $\left\{p_{\alpha}^{I^{l+1}} ; 1 \leqq \alpha \leqq m, I_{l+1}=\left(i_{1}, \cdots, i_{l+1}\right), 1 \leqq i_{1}, \cdots, i_{l+1} \leqq n\right\}$, where $m=\operatorname{dim} M$ $-n$. Then we have the identity

$$
m\left(\begin{array}{c}
l+n \\
l+1
\end{array}\right)=\operatorname{dim} p\left(C_{X_{0}}(\Phi)\right)+\operatorname{rank}\left(\frac{\partial \tilde{\varphi}_{i}}{\partial \tilde{p}_{h}}\right) x_{0} .
$$

We denote by $T$ the rank of the matrix $\left(\frac{\partial \tilde{\varphi}_{i}}{\partial \widetilde{p}_{h}}\right)_{x_{0}}$ and assume that we have the inequality

$$
\frac{D\left(\tilde{\varphi}_{1}, \cdots, \tilde{\varphi}_{T}\right)}{D\left(\tilde{p}_{1}, \cdots, \tilde{p}_{T}\right)} \neq 0
$$

at $X_{0}$.

Every $\tilde{\varphi}_{i}$ can be expressed in the form 


$$
\tilde{\varphi}_{i}=\sum_{j=1}^{K} C_{i}^{j} \tilde{p}_{j}+\psi_{i}, \quad\left(K=m\left(\begin{array}{c}
l+n \\
l+1
\end{array}\right)\right)
$$

where $C_{i}^{j}$ and $\psi_{i}$ belong to $\mathcal{O}_{x_{0}}\left(J^{l}\right)$. By the assumption we have the inequality

$$
\operatorname{det}\left(C_{i}^{j}\left(X_{0}\right)\right)_{i, j=1,2, \cdots, T} \neq 0 .
$$

For every $i(\geqq T+1)$ we can solve the following system of linear equations with unknown functions $B_{i}^{j}(1 \leqq j \leqq T)$ uniquely:

$$
\sum_{j=1}^{T} B_{i}^{j} C_{j}^{k}=C_{i}^{k} \quad(1 \leqq k \leqq T) .
$$

The solutions $B_{i}^{j}$ belong to $\mathcal{O}_{x_{0}}\left(J^{l}\right)$. Then every $\tilde{\varphi}_{i}(i=t+1)$ is expressed in the form

$$
\tilde{\varphi}_{i}=\sum_{j=1}^{T} B_{i}^{j} \tilde{\varphi}_{j}+\sum_{k=T+1}^{K} A_{i}^{k} \tilde{p}_{k}+\phi_{i} .
$$

Here $A_{i}^{k}$ and $\phi_{i}$ are the following functions:

$$
A_{i}^{k}=C_{i}^{k}-\sum_{i=1}^{T} B_{i}^{j} C_{j}^{k} \quad(T+1 \leqq k \leqq K)
$$

and

$$
\phi_{i}=\psi_{i}-\sum_{j=1}^{T} B_{i}^{j} \psi_{j}
$$

The functions $A_{i}^{k}$ and $\phi_{i}$ belong to $\mathcal{O}_{x_{0}}\left(J^{l}\right),(T+1 \leqq i, k)$.

It follows from the assumption (ii) that every $A_{i}^{k}$ vanishes on a neighbourhood of $X_{0}$ in $I \Phi$ and that every $A_{i}^{k}$ belongs to $\Phi_{X_{0}}$. Hence we see that every $\phi_{i}$ belongs to $\left(p_{0} \Phi\right)_{X_{0}}$ by the definition of $p_{0} \Phi$. It follows from assumption (i) that every $\phi_{i}$ belongs to $\Phi_{X_{0}}$.

Let $\mathcal{U}_{0}$ be a sufficiently small neighbourhood of $X_{0}$. Then for every $\widetilde{X}$ in $\left(\rho_{l}^{l+1}\right)^{-1} \bigcup_{0},(p \Phi)_{\tilde{X}}$ is generated by $\varphi_{1}, \cdots, \varphi_{s}$ and $\tilde{\varphi}_{1}, \cdots, \tilde{\varphi}_{T}$. This proves the proposition, because $\sigma_{l}^{l+1} d \tilde{\varphi}_{1}, \cdots, \sigma_{l}^{l+1} d \tilde{\varphi}_{T}$ are independent at $\widetilde{X}$. Here $\sigma_{l}^{l+1}$ is the projection from $T_{\widetilde{X}}^{*}\left(J^{t+1}\right)$ onto $T_{\widetilde{X}^{*}}\left(J^{l+1}\right) / \mathrm{Im}$ $\left(\rho_{l}^{l+1}\right)^{*}$.

Remark. Let $X_{0}$ be an ordinary integral point of $\Phi$ which only satisfies the condition (ii) in Proposition 3. Let us consider the system $\Phi_{0}$ generated by $\Phi$ and $\phi_{i}(T+1 \leqq i \leqq n s)$. Then we have the identity $p_{0} \Phi=\Phi_{0}$ on $\bigcup_{0}$, if $\Phi_{0}=0$ is a regular local equation of 
$I \Phi_{0}$ around $X_{0}$.

\section{§3. Involutive systems of differential equations}

In [10] M. Kuranishi defined involutiveness by the following

Definition 1. We say that $\Phi$ is involutive at $X_{0}$, when the following three conditions (i) (iii) are satisfied:

(i) $X_{\mathrm{c}}$ is an ordinary integral point of $\Phi$.

(ii) There exists a domain $\widetilde{\vartheta}_{0}$ in $J^{l+1}(M, N ; \pi)$ such that $\widetilde{\mathcal{V}}_{0} \cap I(p \Phi)$ is a submanifold $\widetilde{\mathcal{V}}_{0}$ which forms a fibered manifold $\left(\widetilde{C}_{0}, C V_{0} ; \rho_{l}^{l+1}\right)$ with a neighbourhood $C_{0}$ of $X_{0}$ in ID.

(iii) $C_{X_{0}}(\Phi)$ is an involutive subspace of $Q_{X_{0}}\left(J^{l}\right)$.

If $\Phi$ is involutive at $X_{0}$, then $\Phi$ is compatible at $X_{0}$. To prove this fact, let $f$ be a mapping from $N$ to $M$ which satisfies the identities $\pi \circ f=$ indentity and $j_{x_{0}}^{l+1}(f)=\widetilde{X}_{0}$. Here $\widetilde{X}_{0}$ is an integral point of $p \Phi$ which satisfies $\rho_{l}^{l+1} \widetilde{X}_{0}=X_{0}$. We see that such $f$ exists by the condition (ii) in Def. 1. We replace the $f$ in the proof of Prop. 2 by the $f$ thus taken. Then we have the identity (1) by the definition of $\partial_{\neq}^{i} \psi$. This proves our assertion.

An involutive system $\Phi$ is completely integrable at $X_{0}$, if and only if the identity $C_{X_{0}}(\Phi)=\{0\}$ holds. Let $\Phi$ be a system of the first order which is completely integrable at every point in $M$. Then for every point $z$ in $M$, there exists the global solution which passes through $z$ (see [10]). T. Nagano treated in [12] completely integrable systems with singularities.

We define quasi-involutiveness by the following

Definition 2. We say that $\Phi$ is quasi-involutive at $X_{0}$, when the following three conditions (i) ( (iii) are satisfied:

(i) $X_{0}$ is an ordinary integral point of $\Phi$.

(ii) $\operatorname{dim} p\left(C_{X}(\Phi)\right)=$ constant on a neighbourhood of $X_{0}$ in I $\Phi$.

(iii) $C_{X_{0}}(\Phi)$ is an involutive subspace of $Q_{X_{0}}\left(J^{\prime}\right)$.

If $\Phi$ is quasi-involutive at $X_{0}$, then there exists a system $\left\{\phi_{i} ; 1 \leqq\right.$ $i \leqq r\}$ of functions in $p_{0} \Phi$ with the following property: $\Phi$ is involutive at $X_{0}$ if and only if $\Phi$ contains all $\phi_{i}(1 \leqq i \leqq r)$. This fact can be 
proved by the method used in the proof of Prop. 3.

We have a necessary and sufficient condition for a system to be involutive in the following

Theorem 1. Let $\Phi$ be a system of differential equations of order $l$. Then in order that $\Phi$ be involutive at $X_{0}$, it is necessary and sufficient that $\Phi$ is p-closed and quasi-involutive at $X_{0}$.

Proof. Let $\Phi$ be $p$-closed and quasi-involutive at $X_{0}$. Then the conditions (i) and (iii) in Def. 1 are satisfied by the definition of quasi-involutiveness. The condition (ii) in Def. 1 is satisfied by Prop. 3.

The necessity of the conditions will be proved later.

M. Kuranishi showed in [10] that his definition of involutiveness coincides with the classical notion of involutiveness, proving the following theorem:

Let $\Phi$ be a system of differential equations of the first order which is involutive at $X_{0}$. Then $\Phi$ is generated by the following functions $\varphi_{\alpha}^{i}\left(0 \leqq i \leqq n, 1 \leqq \alpha \leqq \kappa_{i}\right)$ in a neighbourhood $\mathcal{U}_{0}$ of $X_{0}$ and $p \Phi$ is generated by $\Phi$ and $\partial_{\sharp}^{r} \varphi_{a}^{2}\left(1 \leqq i \leqq n, 1 \leqq \alpha \leqq \kappa_{i}, 1 \leqq r \leqq i\right)$ in the domain $\left(\rho_{1}^{2}\right)^{-1} \bigcup_{0}$ :

where

$$
\left\{\begin{array}{l}
\varphi_{\alpha}^{0}=y_{\alpha}-\psi_{\alpha}^{0}\left(x, q^{0}\right), \\
\varphi_{\alpha}^{i}=p_{\alpha}^{i}-\psi_{\alpha}^{i}\left(x, y, p^{1}, \cdots, p^{i-1}, q^{i}\right), \\
\left(0 \leqq \kappa_{0} \leqq \cdots \leqq \kappa_{n} \leqq m\right),
\end{array}\right.
$$

$$
x=\left(x_{1}, \cdots, x_{n}\right) \text {, independent variables }
$$$$
y=\left(y_{1}, \cdots, y_{m}\right) \text {, dependent variables }
$$$$
p^{i}=\left(p_{1}^{i}, \cdots, p_{m}^{i}\right) \text {, derivatives with respect to } x_{i}
$$

and

$$
q^{i}=\left(p_{x_{i}+1}^{2}, \cdots, p_{m}^{i}\right), \quad q^{0}=\left(y_{\kappa_{0}+1}, \cdots, y_{m}\right) .
$$

Then we can construct the general solution of $\Phi$ around $X_{0}$ by solving successively equations of Cauchy-Kowalevsky's type.

For a system $\Phi$ of higher order, we construct from $\Phi$ the system of differential equations of the first order $\hat{\Phi}$ in the natural way.

For example, let $\Phi$ be a system of the second order generated by 
where

$$
\varphi_{k}(x, y ; z ; p, q ; r, s, t), \quad(1 \leqq k \leqq r),
$$

$$
\begin{gathered}
x, y ; \text { independent valiables } \\
z \quad ; \text { dependent valiable } \\
p=\frac{\partial z}{\partial x}, \quad q=\frac{\partial z}{\partial y}, \quad r=\frac{\partial^{2} z}{\partial x^{2}}, \quad s=\frac{\partial^{2} z}{\partial x \partial y}, \quad t=\frac{\partial^{2} z}{\partial y^{2}} .
\end{gathered}
$$

Then $\hat{\boldsymbol{\sigma}}$ is the system of the first order generated by

and

$$
\varphi_{k}\left(x, y ; z, p, q ; \frac{\partial p}{\partial x}, \frac{\partial q}{\partial x}, \frac{\partial q}{\partial y}\right), \quad(1 \leqq k \leqq r)
$$

$$
\frac{\partial z}{\partial x}-p, \quad \frac{\partial z}{\partial y}-q, \quad \frac{\partial p}{\partial y}-\frac{\partial q}{\partial x} .
$$

In general $\hat{\Phi}$ has the following properties: integration of $\hat{\Phi}$ is equivalent to that of $\Phi$ and, $\hat{\Phi}$ is involutive if and only if $\Phi$ is involutive. Hence in the general case Kuranishi's definition of involutiveness coincides with the classical notion of involutiveness.

Also $\hat{\mathscr{D}}$ has the following properties (i) and (ii):

(i) $\hat{\Phi}$ is $p$-closed if and only if $\Phi$ is $p$-closed.

(ii) $\hat{\Phi}$ is quasi-involutive if and only if $\Phi$ is quasi-involutive.

Now we shall prove the necessity in Th. 1 . In the case where $\Phi$ is of the first order, we can prove the necessity by Kuranishi's theorem above stated. Hence, in the general case, we see that the conditions in Th. 1 are necessary by the remark above mentioned.

In the case where $\Phi$ is a system of the first order with one unknown function, the subspace $C_{X}(\mathscr{D})$ is always involutive (see [10]). Hence in this case our theorem is equivalent to the classical theorem of Lagrange and Jacobi (see [4], [6], [10]).

\section{\$4. The prolongation theorem}

M. Kuranishi obtained in [9] and [10] the following prolongation theorem:

For every non-negative integer $n$, let $\Phi_{n}$ be a system of differential equations of order $l+n$. We assume that, $p \Phi_{n} \subset \Phi_{n+1}$ for every 
$n$ and that, there exist for every $n$ an ordinary integral point $X_{0}^{n}$ of $\Phi_{n}$ and its neighbourhood $C_{n}$ in I $\Phi_{n}$ which satisfy the following two conditions (i) and (ii):

(i) $\rho_{l+n}^{l+n+1} X_{0}^{n+1}=X_{0}^{n}$,

(ii) $\left.(C\rangle_{0}^{n+1}, C V_{0}^{n} ; \rho_{l+n}^{l+n+1}\right)$ forms a fibered manifold.

Then $\Phi_{n}$ is involutive at $X_{0}^{n}$ for sufficiently large $n$.

In his new proof he applied the following

Lemma. (Kuranishi-Guillemin-Singer-Sternberg-Serre). Let $E$ and $F$ be vector spaces over real numbers. For every non-negative integer $n$, let $A_{n}$ be a linear subspace of $E \otimes S^{l+n}\left(F^{*}\right)$. We assume that $A_{n}$ is contained in $p\left(A_{n-1}\right)$ for every $n(\geqq 1)$. Then for sufficiently large $n$, we have the identity $p\left(A_{n-1}\right)=A_{n}$ and $A_{n}$ is an involutive subspace.

Let $\Phi$ be a system of differential equations of order $l$. We define $p_{0}^{n} \mathscr{\Phi}$ inductively by $p_{0}^{n} \mathscr{D}=p_{0}\left(p_{0}^{n-1} \mathscr{\Phi}\right)$ for every $n \geqq 1$. We call the subsheaf $\bigcup_{n=1}^{\infty} p_{0}^{n} \Phi$ the $p$-closure of $\Phi$. Then the $p$-closure of $\Phi$ is a system of differential equations of order $l$ which is $p$-closed at every point in $J^{\prime}(M, N ; \pi)$. By the definition we see that integration of $\Phi$ is equivalent to integration of the $p$-closure of $\boldsymbol{D}$.

Theorem 2. Let $\Phi$ be a system of differential equations of order l. We define $\Psi_{n}$ inductively as the p-closure of $p \Psi_{n-1}$ for every integer $n$, where $\Psi_{0}$ is the p-closure of $\emptyset$. We assume that there exists an ordinary integral point $X_{0}^{n}$ of $\Psi_{n}$ for every $n$ with the following two properties (i) and (ii):

(i) $\rho_{l+n-1}^{l+n} X_{0}^{n}=X_{0}^{n-1}(n \geqq 1)$,

(ii) $\operatorname{dim} p\left(C_{X}\left(\Psi_{n}\right)\right)=$ constant on a neighbourhood of $X_{0}^{n}$ in $I \Psi_{n}$.

Then $\Psi_{n}$ is involutive at $X_{0}^{n}$ for sufficiently large $n$.

Proof. Since $p \Psi_{n-1}$ is contained in $\Psi_{n}$, it fullows from the Lemma that $C_{X^{n}}\left(\Psi_{n}\right)$ is involutive for sufficiently large $n$. By Theorem 1 we see that the system $\Psi_{n}$ is involutive at $X_{0}^{n}$ for such $n$, because $\Psi_{n}$ is $p$-closed at every point. 
Corollary. Let $\Phi$ be a system of differential equations of order l. We assume that the system $\Phi$ has a solution $f$ defined on a domain $U$ in $N$ and that, there exists a point $x_{0}$ in $U$ with the following properties (i) and (ii):

(i) $X_{0}^{n}=j_{x_{0}}^{l+n}(f)$ is an ordinary integral point of $\Psi_{n}$ for every $n$.

(ii) $\operatorname{dim} p\left(C_{X}\left(\Psi_{n}\right)\right)=$ constant on a neighbourhood of $X_{0}^{n}$ in $I \Psi_{n}$ for every $n$.

Here $\Psi_{n}$ is the system of differential equations of order $l+n$ defined for $\Phi$ in Theorem 2. Then $\Psi_{n}$ is involutive at $X_{0}^{n}$ for sufficiently large $n$.

Example 1. (given by Kuranishi in [8]). Let $\Phi$ be the system generated by the following functions $\varphi_{1}, \varphi_{2}$ and $\varphi_{3}$ :

$$
\begin{aligned}
& \varphi_{1}=u_{1}+x_{2} \frac{\partial u_{1}}{\partial x_{2}}, \\
& \varphi_{2}=u_{2}+x_{1} \frac{\partial u_{2}}{\partial x_{1}}, \\
& \varphi_{3}=\frac{\partial u_{1}}{\partial x_{2}}-\frac{\partial u_{2}}{\partial x_{1}} .
\end{aligned}
$$

The general solution of $\Phi$ is given by

$$
u_{1}=\frac{c}{x_{1}^{2} x_{2}}, \quad u_{2}=\frac{c}{x_{1} x_{2}^{2}},
$$

where $c$ is a constant. The system $\Phi$ can not be prolonged to an involutive system by the standard prolongation. For the system $\Phi$ we see that $\Psi_{0}$ is generated by $\Phi$ and the following functions $\varphi_{4}$ and $\varphi_{5}$ :

$$
\begin{aligned}
& \varphi_{4}=\partial_{\sharp}^{1}\left(x_{1} u_{1}-x_{2} u_{2}\right)=u_{1}+x_{1} \frac{\partial u_{1}}{\partial x_{1}}-x_{2} \frac{\partial u_{2}}{\partial x_{1}}, \\
& \varphi_{5}=\partial_{\sharp}^{2}\left(x_{1} u_{1}-x_{2} u_{2}\right)=x_{1} \frac{\partial u_{1}}{\partial x_{2}}-u_{2}-x_{2} \frac{\partial u_{2}}{\partial x_{2}} .
\end{aligned}
$$

The system $\Psi_{0}$ is involutive at every integral point $X$ for which $x_{1} \neq 0$ and $x_{2} \neq 0$.

Example 2. Let $\Phi$ be the system generated by $\varphi_{1}=y-p^{2}$, where 
$p=\frac{d y}{d x}$. Then $\Psi_{0}=\Phi$, and $p \Psi_{0}$ is generated by $\varphi_{1}$ and $\varphi_{2}=p\left(p^{\prime}-\frac{1}{2}\right)$, where $p^{\prime}=\frac{d^{2} y}{d x^{2}}$. We have

$$
\left(\Psi_{1}\right)_{x}=\left\{\begin{array}{l}
\left\{\varphi_{1}, \varphi_{2}\right\}, \quad\left(p^{\prime}=\frac{1}{2}\right) \\
\left\{y, p, p^{\prime}\right\}, \quad\left(p^{\prime} \neq \frac{1}{2}\right) .
\end{array}\right.
$$

Hence $\Psi_{1}$ is involutive at $(x, 0,0,0)$.

By the standard prolongation the solution $y=0$ remains a singular solution of $p^{n} \Phi$ for every $n$.

Example 3. (Clairaut's equation). Let $\Phi$ be the system generated by $\varphi_{1}=y-p x-f(p)$. Then $p \Phi$ is generated by $\varphi_{1}$ and $\varphi_{2}=p^{\prime}\left(x-f^{\prime}(p)\right)$. We have

$$
\left(\Psi_{1}\right)_{X}=\left\{\begin{array}{l}
\left\{\varphi_{1}, p^{\prime}\right\}, \quad\left(x-f^{\prime}(p) \neq 0\right) \\
\left\{\varphi_{1}, x-f^{\prime}(p), \quad 1-f^{\prime \prime}(p) p^{\prime}\right\}, \quad\left(p^{\prime} \neq 0\right) \\
\left\{\varphi_{1}, \varphi_{2}\right\}, \quad\left(p^{\prime}=x-f^{\prime}(p)=0\right) .
\end{array}\right.
$$

Hence Clairaut's singular solution is a regular solution of $\Psi_{1}$.

Example 4. Let $\Phi$ be the system generated by

$$
\varphi_{1}=z-p x-q y+\frac{1}{2}\left(p^{2}+q^{2}\right),
$$

where $p=\frac{\partial z}{\partial x}, q=\frac{\partial z}{\partial y}$. Then $p \Phi$ is generated by $\varphi_{1}, \varphi_{2}$ and $\varphi_{3}$ :

$$
\begin{aligned}
& \varphi_{2}=r(x-p)+s(y-q), \\
& \varphi_{3}=s(x-p)+t(y-q),
\end{aligned}
$$

where $r=\frac{\partial^{2} z}{\partial x^{2}}, s=\frac{\partial^{2} z}{\partial x \partial y}$ and $t=\frac{\partial^{2} z}{\partial y^{2}}$.

We have

$$
\left(\Psi_{1}\right)_{X}=\left\{\varphi_{1}, p-x, q-y, r-1, s, t-1\right\}
$$

at $X$ for which $r t-s^{2}=0$. Hence Lagrange's singular solution is a regular solution of $\Psi_{1}$. 


\section{BIBLIOGRAPHY}

[1] Bourbaki, N., Éléments de mathématique, algèbre commutative, Chap. 4, Hermann, Paris, 1961.

[2] Cartan, E., Sur certaines expressions différentielles et le problème de Paff, Ann. Sci. Ecole Norm. Sup. 16 (1899), 239-332.

[3] Les systèmes différentielles extérieures et leurs applications géométriques, Hermann, Paris, 1946.

[4] Goursat, E., Leçons sur 1'intégration des équations aux dérivées partielles du premier ordre, deuxième edition. Hermann, Paris, 1921.

[5] Guillemin, V.W. and S. Sternberg, An algebraic model of transitive differential geometry, Bull. Amer. Math. Soc. 70 (1964), 16-47.

[6] Hermann, R., E. Cartan's geometric theory of partial differential equations, Advances in Math. 1 (1965), 265-317.

[7] Johnson, H.H., On terminating prolongation procedures, Pacific J. Math. 10 (1960), 577-583.

[8] Kuranishi, M., On E. Cartan's prolongation theorem of exterior differential systems, Amer. J. Math. 79 (1957), 1-47.

[9] Lectures on exterior differential systems, Tata Institute of Fundamental Research, Bombay, 1962.

[10] - On involutive systems of differential equations, Lectures at Res. Inst. Math. Sci., Kyoto University, 1966, to appear in the Sügaku (Japanese).

[11] - On resolution of partial differential equations, Proceedings of Katata Conference on the theory of partial differential equations and on the theory of complex manifolds, Kyoto (1966), 45-54.

[12] Nagano,T., Linear differential systems with singularities and an application to transitive Lie algebras, J. Math. Soc. Japan 18 (1966), 398-404.

[13] Quillen, D. G., Formal properties of over-determined systems of linear partial differential equations, Thesis, Harvard University, 1964.

[14] Schouten, J.A. and W.v.d. Kulk, Pfaff's problem and its generalizations, Oxford, 1949.

[15] Singer, I.M. and S. Sternberg, The infinite groups of Lie and Cartan, J. Analyse Math. 15 (1965), 1-114.

[16] Spencer, D.C., Deformation of structures on manifolds defined by transitive, continuous pseudogroups, I, II, III, Ann. of Math. 76 (1962), 306-445 and 81 (1965), 389-450.

[17] Some remarks on overdetermined systems of linear partial differential equations, Proceedings of Katata Conference on the theory of partial differential equations and on the theory of complex manifolds, Kyoto (1966), $55-82$.

[18] Sweeney, W.J., The D-Neumann problem, Thesis, Stanford University, 1966.

[19] Tanaka, N., On generalized graded Lie algebras and geometric structures, I, to appear in the J. Math. Soc. Japan. 